\title{
Evaluation of Vaccine Preventable Diseases (Measles and Diphtheria) Surveillance System - Delhi, India, 2013
}

\author{
Kapil Goel* \\ National Center for Disease Control, Delhi, India
}

\section{Objective}

The specific objective was to evaluate the VPD surveillance system of Delhi, focusing on measles and diphtheria.

\section{Introduction}

The vaccine preventable diseases (VPDs) of measles and diphtheria in India were responsible for $47 \%$ of global measles mortality and $20 \%$ of global diphtheria mortality in 2010 . We evaluated the VPD surveillance system of Delhi, focusing on measles and diphtheria.

\section{Methods}

We evaluated the Health Management Information System (HMIS) and reviewed the available data for measles and diphtheria surveillance from North Delhi district from 2012 to 2013. Primary data were collected by interviewing key state and district level stakeholders using a semi-structured questionnaire. Documents like protocols, operational manuals, training documents and hospital and dispensary records were also reviewed.

\section{Results}

HMIS is a web-based reporting system started in Delhi in April 2008. Data are collected through passive surveillance. The system uses standard definitions and reporting mechanisms. Data validation is standardized and documented. The system is useful and simple to use; the system has shown flexibility in adapting to needed changes over time. The system is acceptable due to involvement of field staff in the process. We observed completeness in reporting of forms (93.8\% [845/900]) and systemic support (manpower, infrastructure, funds) for effective functioning of HMIS. The surveillance system is sensitive enough to see trends but data are not available at the population level to know the true burden of disease. The data quality is good for case data but poor for mortality data. The system provides incomplete representation for private sector [captures only 3.3\% of reporting units (15/450)]. Only $42 \%$ (38/90) of reporting units reported on time for January 2013.

\section{Conclusions}

The overall quality of the surveillance system is good, and it is meeting its objectives. However timeliness of reporting and representativeness needs further improvement.

\section{Keywords}

Measles; Diphtheria; Surveillance; Vaccine; System

\section{Acknowledgments}

None

*Kapil Goel

E-mail: drkapil123@gmail.com 